\title{
EL MEDIO AUDIOVISUAL Y DIGITAL COMO VEHÍCULO TRANSMISOR ENTRE EL MUNDO ACADÉMICO Y EL LABORAL EN LA EDUCACIÓN SUPERIOR DE LA SOCIEDAD DEL CONOCIMIENTO
}

\author{
Un estudio de caso
}

The audiovisual and digital media as transmitting vehicle between academic and laboral word in higher education Knowledge Society: A Case Study

\author{
DAVID MARTÍNEZ SÁNCHEZ
}

Universidad de Salamanca, España

\section{KEY WORDS}

Audiovisual

and Digital Media

Knowledge Society

Higher Education

Educommunication

\section{ABSTRACT}

We live in a post -industrial phase in which physical capital and the factory has given way to the information and knowledge as a bargaining chip. But information and knowledge, led by the inclusion of ICTs become insufficient in a third stage of the life of the learner; higher education, not allowing for a direct and real relationship between academic life and working life is sought to undertake in the completion of this stage, which increases in non-technological careers. A new concept is needed; intellection, or manage and adapt that information and knowledge contained in the earlier stages of education to make it a starting point for a real training in the new society in which we live, defined by the audiovisual and digital media.
PALABRAS CLAVE

Medios audiovisuales $y$ digitales

Sociedad del Conocimiento

Educación Superior

Educomunicación

\section{RESUMEN}

Vivimos en una etapa post-industrial en la que el capital físico y la fábrica han dado paso a la información y el saber como moneda de cambio. Pero la información y el conocimiento, lideradas por la inclusión de las TICs, se hacen insuficientes en un tercer estadio de la vida del educando; la educación superior, ya que no permiten realizar una relación directa y real entre la vida académica y la vida laboral que se busca emprender en la finalización de esta etapa, que aumenta en carreras y ciclos superiores no tecnológicos. Es necesario un nuevo concepto; la intelección, o administrar y adaptar esa información y conocimiento recogidas en las anteriores etapas educativas para convertirlo en un punto de arranque para una verdadera formación en la nueva Sociedad en la que vivimos, definida por los medios audiovisuales y digitales. 


\section{Introducción y estado de la cuestión}

$\mathrm{E}$ n los dos últimos siglos de historia, las sociedades globales han sufrido tres cambios radicales y profundos de los cimientos sociales, económicos y tecnológicos. Las principales fuentes de energía, la industria, las formas de comunicación y de desplazamiento de las personas, e incluso el concepto de los recursos más valiosos e imprescindibles para la vida en sociedad han derivado en lo que se han denominado como grandes revoluciones de la humanidad, las dos primeras Industriales y la tercera científicotecnológica (Corona, 1998: 65-66). Pero si las dos primeras revoluciones, situadas cronológicamente en la segunda mitad del Siglo XVIII y la segunda mitad del Siglo XIX respectivamente, trajeron grandes adelantos científicos en forma de nuevas fuentes de energía como la máquina de vapor de agua, el motor de combustión interna o la electricidad, pasar del tren al automóvil o automatizar la que, a lo largo de estos dos períodos, ha sido el principal objetivo social; la industria, la denominada como Tercera Revolución Industrial, o Era Post-industrial, ha sacudido los cimientos de dichos objetivos, pensamientos y sociedad, cambiando el mundo en todos sus ámbitos (Bell, 2001: 8-21). La información y el conocimiento han ocupado el lugar de la industria como principal moneda de cambio. La importancia del capital físico, propia y característica de esta Sociedad Industrial darán paso al capital intelectual dentro de todos los factores de la sociedad, desde la economía, la política o la educación (Rifkin, 2002: 13-18).

La evolución de estos principios ha llevado a la catalogación de Sociedad de la Información, una Sociedad que tiene acceso instantáneo (al menos la mayor parte de ella) a la Información (Cid, Díaz, Moreno, Torres, 2002: 227), y crea un concepto nuevo cuando nos referimos a la misma hablando de Educación, haciendo alarde de la denominada como Sociedad del Conocimiento (Nieto, Rodríguez, 2010: 194). Es en este punto cuando muchos autores muestran su disconformidad en cuanto a la relación de dichos conceptos ya que, mientras algunos de ellos engloba ambos términos como una única realidad (Barroso, 2013; Cabero, 2001; Coll, 2005; Macau, 2005; Maldonado, 2004; TorrentSellens, 2008), en la que el Conocimiento hace alusión al terreno educativo, pero queda englobado dentro de la Información que caracteriza a la actual Sociedad, otros hablan de una evolución a lo largo de las últimas décadas, habiendo existido en un primer momento una Sociedad de la Información que ha dado paso al Conocimiento que define a la Sociedad de hoy en día (Araiza, 2012; Giner, 2004; Martínez, 1999; Pavón, 2010; Rozo, Cardona, 2012).

En lo que todos los autores sí que se ponen de acuerdo es en la aparición y consecuente inclusión de las nuevas Tecnologías de la Información y de la comunicación -TIC o NTIC- como vehículo tanto receptor como transmisor de la información de esta Revolución de la Información y el Conocimiento (Fundación Telefónica, 2014; Macau, 2005; Mas, Quesada, 2005; Recaman, Vargas, 2010), siendo sus principales exponentes internet, englobando el medio audiovisual y el medio digital. El problema reside en que la sociedad ha evolucionado muy rápido, como indica Kurzweil "We often find not just simple exponential growth, but double exponential growth, meaning that the rate of exponential growth is itself growing exponentially" (2005:221222). Y esa velocidad de evolución ha hecho que la sociedad no esté preparada para un cambio inmediato, y sobretodo, tan profundo. El acceso a la información ha cambiado la mentalidad de la sociedad, e cuanto a la comunicación, y la manera de ver las cosas; cambiando los hábitos de consumo, de ocio y tiempo libre y, como no, transformando sustancialmente el mundo laboral, y haciendo que muchos conocimientos desaparezcan si no evolucionan al ritmo de las nuevas necesidades sociales de la actualidad. Consecuentemente, cambiando la forma en que la educación debe ser impartida para adecuarse a este nuevo mundo laboral presidido por los distintos soportes audiovisuales y digitales sobre todo en el tercer estadio de la vida del educando, la educación superior, por ser la más cercana al fin del ámbito académico y al inicio del laboral (VEGA, 2010: 34).

\section{El mundo laboral en la Formación Profesional Superior}

El presente estudio de caso se ha realizado en torno a la formación profesional por dos razones principales. La primera de ellas es la adecuación de la que se busca dotar a estos estudios del salto académico al entorno laboral (Ley Orgánica 5/2002, de 19 de junio, de las Cualificaciones y de la Formación Profesional), y la segunda es la comprobación de familias educativas buscadas en la presente investigación, que siguen la intención de apartarnos de la zona de confort de los estudios tecnológicos. Es decir, postular el objetivo de que el conocimiento del medio es menor cuanto más se aleja de dichos estudios del tipo de Ciencias de la Información, Informática y semejantes.

En cuanto al salto al mundo laboral, es cierto que la Formación Profesional destaca por la búsqueda de ofrecer el incentivo de una exhaustiva preparación y formación para acceder al mismo. Es uno de los principales objetivos, pero también uno de los más importantes lastres que se aplican a estos estudios y que desemboca en una de las principales razones del abandono escolar en esta etapa; la falta de meta (CoboS, Planas, 2012: 55-57; Ruíz, Dussel, 1999: 6-8). Es difícil para un educando comprobar que los estudios realizados no se asemejan al mundo con el que él 
mismo interacciona en su día a día, de forma que pueda acabar con el interés por los mismos. Es cierto que esta etapa educativa no está exenta de prácticas, pero éstas en una gran mayoría de ocasiones se centran en una porción muy limitada del campo estudiado, pudiendo incluso llegar a frustrar al educando en vez de dotarle de conocimientos prácticos.

No se quiere decir que dichas prácticas laborales son insuficientes, y aún menos teniendo en cuenta la actual inclusión de la Formación Laboral Dual que busca un paralelismo entre estudios y trabajo integrado en empresas reales, pero en la que sí que es cierto que, bien por las propias experiencias de los educandos, bien por las diversas empresas que ofrecen dichas prácticas reales, podemos encontrar una correlatividad entre buenas y malas experiencias por parte de los educandos de esta etapa (Macías, 2012).

Es interesante también fijarse en las propias familias educativas, ya que hallamos que en las familias más tecnológicas como las anteriormente mencionadas, se hacen referencias continuas a las Tecnologías de la Información y la Comunicación; a pesar de ser éstas en forma de herramientas para apoyar los recursos teóricos y prácticos y sin profundizar categóricamente en los usos laborales que pueden brindar, pero ¿Qué ocurre en los estudios más alejados de esta rama tecnológica? La Formación Profesional de Grado Superior ofrece la educación de Oficios, como Escultura, Encuadernación o Forja, que corren incluso peligro de desaparición hoy en día si no evolucionan para tener presencia en la actual Sociedad $\mathrm{y}$, como intentaremos igualmente demostrar, las enseñanzas del medio disminuyen en este tipo de familias, quedando reducidas normalmente a la mera acepción de transversalidad $\mathrm{y}$, sobre todo, no realizándose una apreciación real del mundo audiovisual y digital, piedra angular de la evolución social, laboral y, por tanto, educativa.

\section{La educación del medio en España}

Pero ¿Cómo se están tratando estos temas en la educación? Si nos fijamos en las principales leyes educativas de presente ( $\mathrm{y}$, al tiempo del escrito, futura) vigencia en España, la L.O.E. y la L.O.M.C.E., podemos la única aparición tanto del término audiovisual como el de las TIC, que se engloba prácticamente como un mismo término, vemos que su protagonismo se centra, o más bien se limita, a la transversalidad de las mismas como podemos observar en sus artículos 19, 24 y 25 en los cuales aparece exactamente en los mismos términos;
Sin perjuicio de su tratamiento específico en algunas de las materias de la etapa, la comprensión lectora, la expresión oral y escrita, la comunicación audiovisual, las tecnologías de la información y la comunicación y la educación en valoresse trabajarán en todas las áreas.

En el caso único de las TICs, no teniendo en cuenta las aptitudes audiovisuales y/o digitales, la situación cambia, aunque tampoco sustancialmente, convirtiendo el empleo de las TICs en una asignatura opcional en bachillerato en sus artículos 25 y 34bis, amén de apariciones concretas en las que se expresa el promover el conocimiento y uso de las tecnologías de la información y la comunicación, sin realizar ningún apunte, salvo el anteriormente indicado de transversalidad, de cómo llevar a cabo estas acciones. Paralelamente, si nos fijamos en la principal ley de formación audiovisual, los términos referidos al entorno audiovisual y/o digital directamente no se pueden encontrar, y si realizamos una búsqueda minuciosa de la valoración académica de las Tecnologías de la Información y de la Comunicación.

En función de las necesidades del mercado de trabajo y de las cualificaciones que éste requiere, se desarrollarán las ofertas públicas de formación profesional, en cuya planificación ha de prestarse especial atención a la enseñanza de las tecnologías de la información y la comunicación, idiomas de la Unión Europea y prevención de riesgos laborales.

En el punto 4 del Artículo 10 de la ley, referido a las ofertas de formación profesional.

Las ofertas públicas de formación profesional favorecerán la utilización de las tecnologías de la información y la comunicación para extender al máximo la oferta formativa y facilitar el acceso a la misma de todos los ciudadanos interesados.

Y en su disposición adicional tercera.

Áreas prioritarias en las ofertas formativas. Son áreas prioritarias que se incorporarán a las ofertas formativas financiadas con cargo a recursos públicos las relativas a tecnologías de la información y la comunicación, idiomas de los países de la Unión Europea, trabajo en equipo, prevención de riesgos laborales así como aquéllas que se contemplen dentro de las directrices marcadas por la Unión Europea.

Y en cuanto a las disposiciones propias de las Comunidades Autónomas, la situación no cambia sustancialmente; 
Tabla 1.1: Relación de Leyes Educativas Autonómicas.

\begin{tabular}{|c|c|c|}
\hline Comunidad & Ley & Observaciones \\
\hline Andalucía & $\begin{array}{l}\text { Ley } 17 / 2007 \text {, de } 10 \text { de diciembre, de Educación } \\
\text { de Andalucía }\end{array}$ & $\begin{array}{l}\text { Solo se hace mención de las tecnologías de la } \\
\text { información y comunicación, haciendo referencia a } \\
\text { ellas como un derecho del alumnado y una } \\
\text { competencia básica, sin hacer referencia al mundo } \\
\text { laboral, únicamente al área transversal. }\end{array}$ \\
\hline Canarias & $\begin{array}{l}\text { Ley 6/2014, de } 25 \text { de julio, Canaria de } \\
\text { Educación no Universitaria }\end{array}$ & $\begin{array}{l}\text { El medio audiovisual y digital no es mencionado, } \\
\text { únicamente las TIC como medio transversal y en } \\
\text { referencia a una herramienta de adscripción de } \\
\text { centros y comunicación con las familias. }\end{array}$ \\
\hline Cantabria & $\begin{array}{l}\text { Ley } 6 / 2008 \text {, de } 26 \text { de diciembre, de Educación } \\
\text { de Cantabria }\end{array}$ & $\begin{array}{l}\text { En su artículo } 46 \text { se indica Preparar a los alumnos } \\
\text { para la actividad en un campo profesional, } \\
\text { proporcionándoles } \\
\text { las competencias profesionales necesarias para } \\
\text { facilitar su adaptación tanto a las modificaciones } \\
\text { laborales que pueden producirse a lo largo de su vida } \\
\text { como a los cambios tecnológicos y organizativos } \\
\text { que caracterizan el mercado de trabajo, aunque } \\
\text { solo se hace referencia a prácticas en empresas. }\end{array}$ \\
\hline $\begin{array}{l}\text { Castilla-La } \\
\text { Mancha }\end{array}$ & $\begin{array}{l}\text { Ley } 7 / 2010 \text {, de } 20 \text { de julio, de Educación de } \\
\text { Castilla-La Mancha }\end{array}$ & $\begin{array}{l}\text { La referencia se realiza a los valores transversales y } \\
\text { al mundo laboral en relación a las prácticas en } \\
\text { empresas. }\end{array}$ \\
\hline Cataluña & $\begin{array}{l}\text { Ley } 12 / 2009 \text {, de } 10 \text { de julio, de Educación de } \\
\text { Cataluña }\end{array}$ & $\begin{array}{l}\text { Acepción del medio digital como enseñanza para la } \\
\text { competencia para la utilización autónoma y creativa } \\
\text { de los sistemas digitales, así como en su artículo } 21 \\
\text { el derecho del alumnado a ser educado en el discurso } \\
\text { audiovisual, aunque no hace más referencia a los } \\
\text { mismos. }\end{array}$ \\
\hline Extremadura & $\begin{array}{l}\text { Ley } 4 / 2011 \text {, de } 7 \text { de marzo, de Educación de } \\
\text { Extremadura }\end{array}$ & $\begin{array}{l}\text { Referencia directa al medio audiovisual y digital, sin } \\
\text { mención de su relación con el mundo laboral. Se } \\
\text { limita al campo transversal junto con otros ámbitos } \\
\text { como educación en valores o medio ambiente. }\end{array}$ \\
\hline
\end{tabular}

Fuente(s): Boletín Oficial del Estado.

\section{Política educativa europea}

Por su parte, en la Unión Europea, la política educativa es competencia de cada país, pero entre todos se fijan líneas de actuación y objetivos comunes. Y por ello nació en el 2002 el Proceso de Copenhague; una estrategia europea destinada a mejorar el rendimiento, calidad y atractivo de la Formación Profesional en Europa, siendo el última de ellos el comunicado de Brujas, que dicta los pasos a dar para la actual década (2010-2020). Pero nuevamente nos encontramos que, a pesar de enumerar como objetivos principales adaptarse a la evolución del mercado laboral y entender los sectores y aptitudes emergentes o también preparar a la gente para que se adapte al cambio y lo gestione haciendo que adquieran competencias claves, vuelve a reducir esa evolución experimentada en la actual Sociedad de la Información y/o el Conocimiento, al empleo de las Tecnologías de la Información y la Comunicación como meras herramientas para lograr competencias, y no como la necesaria cohesión académico-laboral que pueden brindar.
La educación y la formación profesionales deben ofrecer a los alumnos la oportunidad de ponerse al día en las competencias clave, de complementarlas y de basarse en ellas, sin descuidar por ellos las cualificaciones profesionales. Concretamente, las cualificaciones y competencias en materia de tecnologías de información y comunicación, así como en lenguas extranjeras, resultarán cada vez más decisivas para conseguir y mantener un empleo y para gestionar la vida cotidiana (Comunicado de Brujas, 2010).

Es curioso, en cambio, como a lo largo de los años y de los diversos procesos (Burdeos, 2008) sí se remarca continuamente la búsqueda de la conexión laboral de la que deben hacer gala los estudios superiores, la necesidad de la renovación de dichos estudios para maximizar su utilidad y calidad y la búsqueda creativa de nuevas configuraciones del proceso académico que maximicen el aprovechamiento real de dichos ciclos por parte del educando. 


\section{Estado de la cuestión: La transversalidad}

De esta manera y como ya hemos afirmado, el uso de los presentes contenidos se queda aislado en la mayor parte de las veces en el limitado mundo de la transversalidad. Pero el problema de la misma ha sido estudiado en profundidad por varios autores, $\mathrm{y}$ en multitud de los casos se define que, debido a la no obligatoriedad, la falta de formación del profesorado o la no evaluación formal de los contenidos, dan lugar a que las asignaturas transversales se obvien en gran parte (Soriano, Zapata, González y Muñoz, 2009: 94), de lo que se desprende que si no existe anteriormente una asignatura que sienta las bases para, como mínimo, llegar a adquirir los conocimientos para poner en práctica en ese ejercicio de transversalidad, dicho proceso puede tornarse en inocuo, y generalmente inexistente, para el educando.

Es por tanto en el marco de la idea de la adaptación de la Educación Superior a la nueva Sociedad del Conocimiento y el evitar su mera concepción como transversal, en el que se enmarca el presente estudio de caso, ya que, al ser el medio digital y audiovisual el predominante de un mundo laboral tecnológico y tecnificado, es, o debería ser, consecuencia natural y lógica que en la educación se tenga en cuenta esta materia en sus múltiples etapas educativas, y sobre todo en la de la educación superior al ser la más próxima a esta nueva realidad laboral que nos engloba.

\section{Metodología de la investigación}

\section{Justificación}

Hemos elegido el módulo de "audiovisuales", contextualizado en el título de Técnico superior de Artes Plásticas y Diseño pertenecientes a la familia profesional de artes aplicadas de la escultura. El módulo escogido converge en su currículo las características deseadas;

- Un módulo de un ciclo superior, acotando así la variable de la edad (al menos en cuanto a mínimo) y, al aumentar los parámetros de dificultad y duración, sumados a los anteriores, establece una mejor comparación entre los diversos componentes del grupo de estudio.

- Un ciclo alejado sustancialmente de las familias tecnológicas, mejor manera de comprobar el objetivo del "olvido tecnológico" referente al distanciamiento de dichos estudios, en las cuales no podríamos resaltar datos realistas, al estar las variables contaminadas por el propio interés personal en el medio digital y/o audiovisual.

- Un módulo de segundo año y, a ser posible, un segundo semestre, para así poder asegurar el máximo de conocimientos tanto impartidos al alumnado como buscados por ellos mismos.

- Sin ser de obligado cumplimiento, era interesante buscar un Ciclo cercano a los oficios tradicionales, considerado en ocasiones con riesgo de continuidad, para así poder evaluar la adaptación del mismo a la evolución social, así como enfrentar los currículos oficiales respecto a un curriculum antiguo con uno de actual y/o futura imposición.

- Un módulo eminentemente procedimental, de forma que pudiésemos evitar una prueba final basada únicamente en memorización de datos $\mathrm{y}$, por el contrario, obligásemos a la realización de una prueba subjetiva, más fácil de contrastar con los contenidos procedimentales y actitudinales buscados.

- Un módulo en el que se pueda hacer uso del contenido mínimo audiovisual para la correcta realización del estudio (equipos informáticos, cámaras de vídeo o fotográficas, ordenadores y programas de edición al menos.

Es importante destacar que el presente módulo no corresponde a las enseñanzas mínimas del currículo oficial del Ciclo del cual forma parte. Tras hacer realizado un análisis tanto del currículo actual (L.O.G.S.E.), como el de futura adaptación (L.O.E.) vemos que no consta en los módulos aquí referidos, si no que se trata de una adaptación curricular de la Comunidad Autónoma en la que se ha realizado el estudio y que, de hecho, va a desaparecer en la próxima adaptación curricular a realizar. Este hecho es completamente propicio ya que permite aunar en un módulo todos los requerimientos deseados. Cabe destacar que el mismo contempla la enseñanza básica del proceso de fotografía y de vídeo, así como elementos de sonido, color y diversas técnicas sencillas de realización, de forma autónoma por los alumnos.

\section{Objetivos e hipótesis}

La hipótesis principal que hemos perseguido en la realización de este estudio es; la necesidad de una asignatura en la educación superior, en este caso en la formación profesional de grado superior, como elemento vehicular para poder conectar el mundo académico y el laboral, a través del medio audiovisual y digital.

En la consecución de la tesis citada, planteamos los siguientes objetivos;

- Comprobar el grado de alfabetización digital de los educandos de familias alejadas de las tecnológicas.

- Comprobar la evolución de los currículos adyacentes a los estudios de oficios. 
- Demostrar la importancia del conocimiento mediático para los educandos en la educación superior.

- Implantar un sistema educativo basado en proyectos, que fomente tanto la autonomía individual como la labor grupal, sobre una educación basada en el aprendizaje y eminentemente conceptual e individualista.

\section{Diseño del estudio}

Para realizar el estudio se ha realizado un trabajo de observación durante el primer semestre y se ha impartido el segundo semestre del módulo elegido, cambiando sustancialmente los contenidos de forma que sirviesen a la hipótesis perseguida sin cambiar los objetivos del mismo. Para ello se ha elegido un proyecto de enseñanza del medio basado en proyectos y se ha implementado una enseñanza de tipo individual primero y colaborativo posteriormente.

Se ha elegido el diseño de estudio de caso debido a las posibilidades de recogida de información que nos ofrecía dicha metodología; where substantial complexity is present in the system being studied, the case method is a valid approach, and possibly the most valid approach available, for exploring and learning about it. (Gill: 49). La investigación realizada es de corte de investigación-acción fundamentalmente, y prevé una recogida de datos tanto cualitativa, mediante el estudio en profundidad de historias de vida, como cuantitativo, mediante la realización de dos test de preguntas cerradas y el estudio de factores cuantitativos referentes como la asistencia o los resultados académicos, realizados ambos a través de una metodología de observación participante.

\section{La muestra}

Para poder condensar todas las características idóneas para la realización del presente estudio, es necesario realizar un muestreo no probabilístico intencional, puesto que hemos elegido el módulo en el que queremos que se centre nuestra investigación, y la muestra serán consecuentemente los alumnos participantes del mismo.

\section{Recogida de información}

Los datos necesarios para poder realizar este estudio se han dividido en tres apartados, dos de ellos de carácter cuantitativo y un tercero cualitativo. Se ha realizado de esta manera para poder recabar datos en cuanto a procesos conceptuales, procedimentales y conceptuales y de esta forma llegar a una serie de conclusiones lo más completas posible.
En un primer lugar se realizará, a modo de fichas de alumnos, un test de conocimientos previos con preguntas básicas sobre alfabetización digital y conocimiento del medio, que será complementado con un pequeño test realizado el primer día para comprobar el conocimiento por parte de los educandos del mercado laboral relacionado con su campo de trabajo, así como las nuevas técnicas de innovación y visualización digital de sus trabajos que pueden utilizar.

Posteriormente se realizará un currículo adaptado del módulo, como ya se ha indicado basado en proyectos y basado en un entorno colaborativo. Es importante comprobar en este punto la reacción actitudinal del alumno acostumbrado al trabajo individual. Para ellos se ha puesto en marcha una serie de estrategias educativas que comprenden;

- Clases magistrales: Se ha utilizado la clase magistral básica, necesaria en algunos contenidos, y la basada en hipótesis, que consiste en el realizar una afirmación de la que los discentes deben autentificar su veracidad.

- Clases de problemas y ejemplos y demostraciones: Los estudiantes aprenden más trabajando sobre el problema que viendo cómo se realiza.

- Juego de roles: se basa en que los alumnos representan un papel delante de sus compañeros.

- Proyecto: el profesor no explica, más bien supervisa cada semana las reuniones en el curso de las cuales los estudiantes van avanzando con su trabajo de equipo. Para la realización del proyecto los estudiantes tienden a alcanzar los objetivos del curso, cumpliendo, a lo largo del semestre o del año, el encargo que les ha sido confiado.

En este apartado se tendrán en cuenta los resultados obtenidos y contrastados con el primer semestre, así como con otros años anteriores tales como el resultado académico, la asistencia diaria y la colaboración y participación en el aula.

Por último se realizará un nuevo test que combinará conocimientos del medio y apreciaciones cualitativas tanto del proceso de enseñanzaaprendizaje, del papel del educador, así como de la apreciación general del semestre y posibles puntos negativos $\mathrm{y} / \mathrm{o}$ a mejorar mediante una última pregunta abierta.

\section{Análisis de datos}

El análisis de los datos se ha realizado de la siguiente forma:

Análisis descriptivo de la información registrada en el primer test realizado a los educandos, así como en la primera clase. Estos han sido resumidos 
en tablas con la finalidad de poder describir la muestra en su totalidad.

Los resultados formales obtenidos (asistencia, calificaciones globales y participación) han sido comparados con los datos normativos y se han sintetizado en una tabla.

La información obtenida en los test finales se ha resumido en dos apartados; se ha vuelto a realizar un análisis descriptivo de la información registrada en las preguntas cerradas de carácter cuantitativo y por otro lado se han resumido los aspectos cualitativos del test de forma comprensiva y se han realizado tablas con los datos obtenidos en referencia a: estimación del educador por parte de los educandos, proceso de enseñanza-aprendizaje, estimación personal de los resultados obtenidos y un pequeño resumen de historias de vida en el que se relatan puntos positivos, negativos y a mejorar en futuras interacciones.

\section{Resultados de la investigación}

Nuestra muestra se ha configurado en un grupo de 14 alumnos cuyas edades oscilan entre los 19 y los 54 años ( $\mathrm{X}=31,57$ D.T.=11,82), un $21 \%$ de los educandos admite no tener conocimiento alguno del medio digital, mientras un 57\% admite poseer conocimientos pero bastante bajos. Es importante comprobar que, igualmente, un 36\% admite no poseer internet en el domicilio, resultados que coinciden en su totalidad con el conocimiento nulo del medio. La antigüedad de los equipos demuestra no tener relación con los conocimientos expresados.

Un punto muy importante converge en el hecho de que, al menos a nivel teórico, no notamos apreciación alguna de la brecha generacional propiciada por la diferenciación de edad y marcada por los llamados nativos digitales en contraposición a los inmigrantes digitales.

Tabla 3.1: Relación de educandos estudiados.

\begin{tabular}{|l|l|l|l|l|l|}
\hline & Edad & Conocimiento del medio & ¿Tiene ordenador? & Antigüedad del Ordenador & ¿Tiene internet? \\
\hline$W 1$ & 19 & Bajo & $\mathrm{Si}$ & 4 Años & NO \\
\hline$W 2$ & 29 & Ninguno & $\mathrm{Si}$ & 1 Año & NO \\
\hline$W 3$ & 48 & Ninguno & $\mathrm{No}$ & - & NO \\
\hline$W 4$ & 20 & Bajo & $\mathrm{Si}$ & $\mathrm{Si}$ Años & S Años \\
\hline$W 5$ & 24 & Bajo & $\mathrm{Si}$ & 2 Años & SI \\
\hline$W 6$ & 23 & Bajo & $\mathrm{Si}$ & 8 Años & NO \\
\hline$W 7$ & 49 & Bajo & $\mathrm{Si}$ & 2 Años & SI \\
\hline$W 8$ & 22 & Medio & $\mathrm{Si}$ & 2 Años & SI \\
\hline$W 9$ & 24 & Medio & $\mathrm{Si}$ & +10 Años & SI \\
\hline$W 10$ & 54 & Bajo & $\mathrm{Si}$ & 2 Años & SI \\
\hline$W 11$ & 22 & Alto & $\mathrm{Si}$ & 5 Años & SI \\
\hline$W 12$ & 42 & Bajo & $\mathrm{Si}$ & +10 Años & SI \\
\hline$W 13$ & 26 & Bajo & $\mathrm{Si}$ & +10 Años & SI \\
\hline$W 14$ & 40 & Ninguno & & NO \\
\hline
\end{tabular}

Fuente(s): Propia.

Los educandos que no tienen internet sí que, en su mayoría, afirman utilizarlo bien en establecimientos preparados para ello, como en casas de amigos, conocidos o familiares y en el propio centro. El uso que se le da al medio digital se distingue claramente por estar dirigido al terreno del ocio (redes sociales, foros, compras, juegos online y offline), adquiriendo una diferencia con los terrenos académico (realización de trabajos, búsqueda de datos en internet, foros y redes académicas $\mathrm{y} / \mathrm{o}$ contacto con el profesorado) $\mathrm{y}$ laboral (redes profesionales, videobook, videocurrículum, videos profesionales, búsqueda activa de empleo). Los resultados se obtuvieron mediante una encuesta con respuestas de escala de calificación, o escala de Likert, de 5 posiciones, a saber: prácticamente cada día, una o dos veces por semana, una o dos veces por mes, nunca o casi nunca. Los resultados nos trasladan que el ocio es el terreno más utilizado, seguido por el académico, dejando un uso laboral olvidado prácticamente en el olvido.

Tabla 3.2: Uso del medio digital y audiovisual.

\begin{tabular}{|l|l|l|l|l|}
\hline $\begin{array}{l}\text { Uso del } \\
\text { Medio digital }\end{array}$ & $\begin{array}{l}\text { Cada } \\
\text { día }\end{array}$ & $\begin{array}{l}\text { Cada } \\
\text { Semana }\end{array}$ & $\begin{array}{l}\text { Cada } \\
\text { mes }\end{array}$ & $\begin{array}{l}\text { Casi } \\
\text { Nunca }\end{array}$ \\
\hline Ocio & $100 \%$ & $0 \%$ & $0 \%$ & $0 \%$ \\
\hline Académico & $57 \%$ & $29 \%$ & $14 \%$ & $0 \%$ \\
\hline Laboral & $0 \%$ & $7 \%$ & $7 \%$ & $86 \%$ \\
\hline
\end{tabular}

Fuente(s): Propia.

Al consultar las razones del limitado uso del medio digital y audiovisual en el entorno laboral, las razones más argumentadas han sido el desconocimiento de sus posibles usos, seguidos por la creencia de la no validez de los citados medios con respecto al campo estudiado y, en un pequeño porcentaje, la limitación técnica de los estudiantes. 
Tabla 3.3: Razones del poco uso del medio digital.

\begin{tabular}{|l|c|c|c|c|}
\hline $\begin{array}{l}\text { Razones del poco uso del medio como } \\
\text { herramienta laboral }\end{array}$ & $50 \%$ & Desconocimiento validez en mis estudios & No sémo hacerlo & Otros \\
\hline
\end{tabular}

Fuente(s): Propia.

En las primeras sesiones se tuvo especial consideración en la explicación de las posibilidades del medio digital y/o audiovisual tanto con el entorno laboral en general, como con su especialidad en particular. Cabe destacar que, en un principio, muchos alumnos no entendían dicha relación y se mostraban muy reacios a cualquier actividad "tan larga y difícil". Se tomó la decisión de convertir el proyecto en la realización desde el primer punto de un cortometraje (creación y desarrollo de la historia, storyboard, organización de las sesiones de grabación, montaje y exposición del trabajo), y después se realizó la comparativa del trabajo realizado con la creación de videocurriculums, videobooks y múltiples proyectos audiovisuales que les pudiesen ayudar en su futuro profesional. Los resultados en cuanto a las calificaciones no difirieron en cuanto a sus aspectos formales, aunque si en cuanto al esfuerzo colaborativo y participativo para lograrlas, aumentando además la asistencia (que descendía alarmantemente en época de exámenes), que incluso propició que los propios discentes pidieran transformar clases de tutoría en clases de refuerzo para realizar el proyecto. En los resultados obtenidos se comprueba la diferenciación con el primer semestre.

Tabla 3.4: Evolución de las actitudes de los educandos.

\begin{tabular}{|l|l|l|l|}
\hline & $1^{o}$ semestre & $2^{o}$ semestre & Evolución \\
\hline Aprobados & $85 \%$ & $92 \%$ & $+7 \%$ \\
\hline Participación & $40 \%$ & $85 \%$ & $+45 \%$ \\
\hline Asistencia & $70 \%$ & $98 \%$ & $+28 \%$ \\
\hline
\end{tabular}

Fuente(s): Propia.

En el test final de conocimientos realizado podemos observar la progresión de todos los alumnos en general. Es este apartado podemos observar un dato referido a la brecha generacional de la que hablábamos anteriormente. Si bien en un test anterior no podíamos percibir una diferencia notable de dicha brecha, propiciado seguramente por haber realizado el estudio en un entorno cuyos integrantes no mostraban un gran interés en la tecnología, al realizar el test último sí que hemos podido notar dicha diferencia en forma de evolución de los conocimientos. Los conocimientos adquiridos, a pesar de no mostrar diferenciación en cuanto a los contenidos teóricos, sí ha demostrado ser diferente en cuanto a los contenidos prácticos, seguramente por la mayor familiaridad de los discentes nativos digitales con el medio que los rodea (smartphone, ordenadores, tablets, etc.), aunque a veces sea inconscientemente.
Tabla 3.5: Evolución de las aptitudes de los educandos.

\begin{tabular}{|l|l|l|l|}
\hline & EDAD & $\begin{array}{l}\text { TEST } \\
\text { PRINCIPIO }\end{array}$ & TEST FINAL \\
\hline$W 1$ & 19 & Bajo & Medio \\
\hline$W 2$ & 29 & Ninguno & Medio \\
\hline$W 3$ & 48 & Ninguno & Bajo \\
\hline$W 4$ & 20 & Bajo & Alto \\
\hline$W 5$ & 24 & Bajo & Medio \\
\hline$W 6$ & 23 & Bajo & Alto \\
\hline$W 7$ & 49 & Bajo & Bajo \\
\hline$W 8$ & 22 & Medio & Medio \\
\hline$W 9$ & 24 & Medio & Alto \\
\hline$W 10$ & 54 & Bajo & Bajo \\
\hline$W 11$ & 22 & Alto & Alto-Muy Alto \\
\hline$W 12$ & 42 & Bajo & Medio \\
\hline$W 13$ & 26 & Bajo & Alto \\
\hline$W 14$ & 40 & Ninguno & Bajo \\
\hline
\end{tabular}

Fuente(s): Propia.

Las conclusiones en cuanto a la visión de los educandos del proceso educativo, la asignatura y los conocimientos adquiridos por los estudiantes han sido excepcionalmente positivos. Es necesario realizar más estudios ya que la predisposición del alumnado (sobre todo una vez explicada la utilidad de la asignatura y afianzada una meta para la misma) ha sido de una acogida excelente, y es necesario comprobar si se ha tratado de un caso aislado o se puede generalizar. Pero por los resultados recopilados en el estudio, los principales problemas son el desconocimiento de las posibilidades que ofrece el medio audiovisual y/o digital, el pensamiento de invalidez y el no entendimiento de la razón por la que impartir dichos conocimientos y, cabe reseñar que una vez sobrepasados esos problemas, la evolución del proceso aumenta enormemente. La siguiente escala de Likert, con cuatro puntos de medición (muy mal, mal, bien, muy bien), demuestran dichos resultados positivos.

Tabla 3.6: Evaluación formal del proceso por parte de los educandos.

\begin{tabular}{|l|c|c|c|c|}
\hline & Muy mal & Mal & Bien & Muy bien \\
\hline $\begin{array}{l}\text { Proceso } \\
\text { educativo }\end{array}$ & $0 \%$ & $0 \%$ & $5 \%$ & $95 \%$ \\
\hline Asignatura & $0 \%$ & $0 \%$ & $3 \%$ & $97 \%$ \\
\hline $\begin{array}{l}\text { Conocimientos } \\
\text { adquiridos }\end{array}$ & $0 \%$ & $1 \%$ & $9 \%$ & $90 \%$ \\
\hline
\end{tabular}

Fuente(s): Propia. 
Y como ya hemos comentado, se ha dejado un espacio abierto para la recopilación de resultados cualitativos, dejando una pregunta abierta de carácter valorativo del proceso y la asignatura. Se dejan algunas de las valoraciones tal y como se recibieron, no sin antes realizar una pequeña discusión final de las mismas. Y es que dejando de lado la actitud del educador que es sumamente importante, y las características del proceso de enseñanza - aprendizaje, que deben evolucionar tanto o más que los contenidos para adecuarse a las características de la actual Sociedad del Conocimiento; los educandos han agradecido en primer lugar que se les explique la razón del presente estudio y, una vez han asimilado la validez del mismo, así como la importancia y usabilidad que se desprende de las enseñanzas impartidas, se ha podido comprobar que han aumentado exponencialmente el interés, el esfuerzo y la atención. Se indica a continuación algunas de las 14 valoraciones recibidas que tienen relación con lo expuesto.

Tabla 3.6: Evaluación actitudinal del proceso por parte de los educandos.

"Si tengo en cuenta que es la primera vez que hago algo similar
y que hasta ahora nunca me había atraído la cultura
audiovisual, tengo que reconocer que lo que a priori parecía
solo un trabajo para una asignatura se ha convertido en todo un
reto. Y este reto me ha producido expectación en cuanto que me
ha ilusionado participar en un trabajo de grupo divertido y eso
ha provocado que ponga todo mi interés en hacerlo lo mejor
posible, y no tanto por los resultados sino por vivir el proceso
creativo con mis compañeros".
"La verdad es que he aprendido mucho con este trabajo, y con
toda la asignatura. Me hubiese gustado tener mucho más
tiempos para dedicarle más horas y que durase más, se me ha
hecho muy corto el curso y hubiese estado bien unas cuantas
clases más".

"Estoy contento con el proceso para hacer el corto, algo más que hemos aprendido. Esto viene bien para el día de mañana".

"Explicación y realización de lo explicado, bien pero muy poco tiempo... Me hubiera gustado haber aprendido aún más ya que es un tema que me ha gustado mucho".

"Mi valoración es muy positiva por la participación y por el entusiasmo que nos ha transmitido el profesor, y por lo que hemos aprendido de manera fácil, cómoda y divertida".

"Propuesta de mejora ninguna. En cuanto a la satisfacción, estoy contento, he aprendido y me he divertido".

"Estoy completamente de acuerdo con los métodos, contenidos y resultado final. El profesor ha motivado a los alumnos y no se ha limitado a la labor puramente docente".

Fuente(s): Propia.

\section{Conclusiones}

Las asignaturas referidas con la comunicación siempre han requerido de aprendizajes constructivos (comprender y generar significados), en los que el proceso de enseñanza - aprendizaje debe ser continuo (Pozo, 2006); en su ámbito comunicativo, precisan de un elemento continuador en el desarrollo de los conocimientos que imparte (Kaplún, 1998), en su ámbito procedimental precisan de un modelo evaluativo por competencias (Cano, 2008) y en su ámbito más relacionado con las nuevas tecnologías, hacen necesaria la presencia de un sistema continuo de evaluación (Delgado y Oliver, 2006). Estos elementos han demostrado combinarse entre sí previniendo la deserción escolar de la asignatura, que ha bajado drásticamente hasta los mínimos de la misma.

En los modelos de evaluación basados en el aprendizaje, no solamente no se tienen en cuenta en la evaluación elementos propios del rendimiento académico, como las actitudes, motivación, etc. (Arias y otros, 2010), sino que no se conciben como parte del proceso (Lafranceso, 2005). Cuando hablamos de educación basada en proyectos y educación colaborativa, vemos que no solo los conocimientos y los procedimientos son merecedores de estudio, si no que todas estas variantes propias del rendimiento del alumnado se complementan como imprescindibles, teniéndose presentes a la hora de la exposición de los resultados globales, ya que no se puede concebir la evaluación por separado (Cano, 2008). De esta forma hemos podido observar que dicho modelo basado en proyectos ha demostrado ser una herramienta altamente eficaz en cuanto a autonomía, trabajo en equipo, aprendizaje constructivo y rendimiento académico en unos estudiantes acostumbrados a trabajar de forma individualista.

La brecha generacional (Martín-Barbero, 1999: 16-19), la distinción entre los inmigrantes digitales $\mathrm{y}$ aquellas personas que han nacido en una Sociedad tecnificada existe. No solamente en cuanto a empleabilidad y manejo de los medios audiovisuales y digitales, sino también en la velocidad de aprendizaje de los mismos. Una de las características de los inmigrantes digitales ha sido el crecimiento dentro de una Sociedad destecnificada, lo cual es un elemento a tener en cuenta en base a futuras referencias. Aquellos estudiantes que, a pesar de no tener demasiado interés en el medio digital y/o audiovisual, han nacido y crecido rodeados del mismo, demuestran un aprendizaje autónomo más eficiente y resuelto.

Se ha podido comprobar que uno de los principales problemas que arrastraba la asignatura en su revisión con la metodología y los resultados obtenidos en anteriores años trataba una de las principales causas de la deserción escolar indicadas por la Unión Europea; la falta de meta. El tratarse de una asignatura que se desviaba sustancialmente de los contenidos comprendidos en el ciclo, tenía un efecto negativo en los educandos. Podemos comprobar que es necesario establecer una conexión real entre el mundo educativo y el mundo laboral; pero que, una vez realizada correctamente 
y asimilada por los educandos, se convierte en un punto focal para los alumnos, que experimentan un aumento de interés, esfuerzo, asistencia y colaboración. Es necesaria, por tanto, una nueva relación educador - educando en la que se elimine de raíz la unidireccionalidad, convirtiéndose el educador en una herramienta que, mediante un trato bidireccional, se configure como mediador entre los alumnos y los conocimientos.

La autonomía y confianza depositada en los alumnos, combinada con la supervisión indirecta, resulta en un recurso de creatividad, originalidad e interés que mediante la continua supervisión directa y la limitación es negada en su práctica totalidad. La atención a los educandos se debe realizar de forma general pero a la vez individualizada, ya que cada alumno es distinto, posee distintas características, virtudes y defectos. El estudio realizado ha demostrado ser altamente eficaz a la hora de realizar este proceso. Al dotar de autonomía al educando y aplicar el aprendizaje basado en proyectos, cada uno de los alumnos ha respondido absorbiendo los conocimientos y adaptándolos a sus propias características para llegar exitosamente al objetivo fijado.

Muchos de los alumnos (en torno al 70\%) han encontrado una vía adicional en cuanto a conocimiento del mensaje y uso del medio para sus propios fines, tanto creativos como laborales. Los recursos obtenidos a lo largo del semestre en que se ha realizado el estudio han servido para variar en algunos casos el proyecto final a realizar, adaptación a los intereses personales profesionales del medio digital mediante los recursos audiovisuales disponibles, e incluso ha supuesto el deseo de continuar la formación dentro del mundo digital y audiovisual para ponerlo en práctica con el campo artístico estudiado, buscando proyectos creativos personales.
La educación superior supone uno de los mayores retos que debe afrontar el educando en su vida académica y por ello debe ser uno de los factores más sensibles que se deben tener en cuenta. Proyectos educativos como la Formación Profesional Dual, nacida en Alemania, ayudan a mejorar este gran paso a realizar, pero en muchas ocasiones se torna en insuficiente. En casos en los que el alumno aúne las condiciones de querer dirigir su camino profesional hacia la empresa en la que realizará las prácticas, suponiendo un sistema idóneo, la contratación por parte del mismo en dicha empresa constituiría un sistema educativo ideal. Pero mientras la realidad no sea así de eficiente, debemos, como mínimo, formar a los educandos no solo en una empresa en particular, sino en una Sociedad en general. La Sociedad del Conocimiento actual, para la que deben ser instruidos los educandos de hoy en día, se caracteriza por el predominio del medio digital y audiovisual, tanto si hablamos de medios de comunicación, como de empleabilidad o de discurso. Por eso es imprescindible que el educando conozca la forma de utilizar estos recursos en su propio beneficio ya que deberá tener disponibles todas las opciones a su servicio a la hora de dar el salto al mundo laboral actual.

\section{Agradecimientos}

Este trabajo de investigación se ha realizado dentro del Programa de Doctorado en Formación en la Sociedad del Conocimiento de la Universidad de Salamanca y bajo la dirección del Doctor Francisco Javier Frutos Esteban y la Doctora Isabel M. Barrios Vicente. 


\section{Referencias}

Araiza, V. (enero-junio 2012). Pensar la sociedad de la información/conocimiento. Biblioteca Universitaria, 15 (1), 35-47. Universidad Nacional Autónoma de México. Recuperado de: http://www.redalyc.org/pdf/285/28528264004.pdf

Barroso, C. (2013). Sociedad del conocimiento y entorno digital. Teoría de la Educación: Educación y Cultura en la Sociedad de la Información. 14(3), 61-86 Recuperado de: http://campus.usal.es/ revistas trabajo/index.php/revistatesi/article/view/11351/11783

Bell, D. (1976 imp. 2001). El advenimiento de la Sociedad Post-Industrial: un intento de prognosis social. Madrid: Alianza.

Cabero, J. (2001). La Sociedad de la Información y el Conocimiento, transformaciones tecnológicas y sus repercusiones en la educación. En Blázquez, F. Sociedad de la Información y Educación. Mérida: Junta de Extremadura, Consejería de Educación, Ciencia y Tecnología.

Cid, J.; Díaz, F.; Moreno, A.; Torres, L. (2002). Procesado de información en comunicaciones. En Figueiras, A. R. Una panorámica de las Telecomunicaciones. Madrid: Pearson Educación.

Cobos, A.; Planas, J.A. (2012). Orientación profesional adaptada a diferentes colectivos y destinatarios: principios de intervención y estrategias. En Planas, J.A. (Coord); Cobos, A.; Gutiérrez-Crespo, E. La orientación profesional y la búsqueda de empleo: experiencias innovadoras y técnicas de intervención que facilitan la inserción laboral. Barcelona: Grao.

Coll, C. (2005). Lectura y alfabetismo en la sociedad de la información. UOC Papers. 1. UOC. Disponible en: http://www.uoc.edu/uocpapers/1/dt/esp/coll.pdf ISSN 1885-1541

Corona, L. (1998). Enfoques económicos de la tecnología. Problemas y perspectivas. En LARA, F. Tecnología: Concepto, Problemas y Perspectiva. México: Siglo XXI.

Fundación TELÉFONICA. (2014). La Sociedad de la Información en España 2013. Barcelona: Ariel.

Gill, G. (2011). Informing with the Case Method: A Guide to Case Method Reserach, Writing \& Faciliation. California: Informing Science.

Giner, F. (2004). Los Sistemas de Información en la Sociedad del Conocimiento. Madrid: ESIC.

Kurzweil, R. (2005). The singularity is near: When humans transcend biology. USA: Penguin.

Ley 12/2009, de 10 de julio, de Educación de Cataluña. Boletín Oficial del Estado, 6 de Agosto, Número 189, página 67041.

Ley 17/2007, de 10 de diciembre, de Educación de Andalucía. Boletín Oficial del Estado, 23 de Enero de 2008, número 20 , página 4467 .

Ley 4/2011, de 7 de marzo, de Educación de Extremadura. Boletín Oficial del Estado, 23 de Marzo de 2011, número 70, página 30994 .

Ley 6/2008, de 26 de diciembre, de Educación de Cantabria. Boletín Oficial del Estado, 24 de Enero de 2009, número 21, página 8214.

Ley 6/2014, de 25 de julio, Canaria de Educación no Universitaria. Boletín Oficial del Estado, 1 de Octubre de 2014, número 238, página 77321.

Ley 7/2010, de 20 de julio, de Educación de Castilla-La Mancha. Boletín Oficial del Estado, 13 de Octubre, Número 248 , página 86359.

Ley Orgánica 2/2006 de 3 de mayo, de Educación. Boletín Oficial del Estado, 4 de Mayo de 2006, número 106, página 17158.

Ley Orgánica 5/2002, de 19 de junio, de las Cualificaciones y de la Formación Profesional. Boletín Oficial del Estado, 20 de Junio de 2002, número 147, página 22437.

Ley Orgánica 8/2013, de 9 de diciembre, para la mejora de la calidad educativa. Boletín Oficial del Estado, 10 de Diciembre de 2013, número 295, página 97858.

Macau, R. (2005). La base tecnológica de la sociedad del conocimiento. En Tuvella, I; Vilaseca, J (coords). Sociedad del Conocimiento: Cómo cambia el mundo ante nuestros ojos. Barcelona: UOC (Universitat Oberta de Catalunya).

Macías, E.Y. (2012). Significado de las prácticas profesionales. La experiencia de un grupo de alumnos de nutrición de la Universidad Guadalajara Lamar. Revista Iberoamericana de Educación. 59/3. Disponible en: http://www.rieoei.org/deloslectores/4769Macias.pdf

Maldonado, C. E. (2004). Universidad e investigación: una relación poco evidente. En Acosta, F. (Coord.). La Política Universitaria en la Sociedad del Conocimiento. Bogotá: Cooperativa editorial magisterio.

Martín-BArbero, J. (1999). Retos culturales de la comunicación a la educación. En Pérez, G.J. et al. Comunicación, Educación y Cultura. Santa Fe de Bogotá: Universidad Pontificia Javeriana.

Martínez, J. (1999). El salto desde la Gestión de la Información hasta la Gestión del Conocimiento. Representación y organización del conocimiento, 5 (1), págs. 41-54. Disponible en: http://ibersid.eu/ojs/index.php/scire/article/view/1105/1087

Mas, M.; Quesada, J. (2005). Las nuevas tecnologías y el crecimiento económico en España. Bilbao: Fundación 
BBVA.

Nieto, S.; Rodríguez, M. J. (2010). Investigación y evaluación educativa en la Sociedad del Conocimiento. Salamanca: Universidad de Salamanca.

Pavón, A. (2010). La supervisión educativa para la Sociedad del Conocimiento. Madrid: La Muralla.

Real Decreto 1843/1994, de 9 de septiembre, por el que se establecen los títulos de Técnico superior de Artes Plásticas y Diseño pertenecientes a la familia profesional de artes aplicadas de la escultura y se aprueban las correspondientes enseñanzas mínimas. Boletín Oficial del Estado, 25 de Octubre de 1994, número 255, página 33162 .

Real Decreto 218/2015, de 27 de marzo, por el que se constituye la familia profesional artística de Escultura, se establece el título de Técnico Superior de Artes Plásticas y Diseño en Técnicas Escultóricas perteneciente a dicha familia profesional artística y se fija el correspondiente currículo básico. Boletín Oficial del Estado, 23 de Abril de 2015, número 97, página 35257.

Recaman, A.; Vargas, I. (2010) La administración educativa: Gestión de las nuevas economías. En Nieto, S.; Rodríguez, M. J. (COORD.). Investigación y evaluación educativa en la sociedad del conocimiento. Salamanca: Universidad de Salamanca.

Rifkin, J. (2022). La era del acceso: la revolución de la nueva economía. Barcelona: Paidós Ibérica.

Rozo, F. E.; Cardona, D. F. (2012). ¿Sociedad del conocimiento o de la información como condicionante en la dirección? Criterio Libre, 10 (16), 259-268. Disponible en: http://www.dialnet.unirioja.es/descarga/articulo/3966901.pdf

Ruíz, C.; Dussel, E. (1997). El reto de la educación superior en la sociedad del conocimiento. México: ANUIES.

Soriano, E.; Zapata, R M; González, A. J.; Muñoz, M. J. (2009). Las TICs y las enseñanzas E y B-Learning en el Master oficial de sexología. Almería: Universidad de Almería.

Torrent-Sellens, J. (2008). TIC, conocimiento y actividad económica: hacia la economía del conocimiento. En Berumen, S.; Arriaza, K. (Coord.). Evolución y desarrollo de las TIC en la economía del conocimiento. Madrid: Ecobook - Editorial del Economista.

Vega, L. (2010). El proceso de Bolonia y la educación comparada. Miradas críticas. Salamanca: Universidad de Salamanca. 